\title{
Erratum to: Using the Cross-Correlation Function to Evaluate the Quality of Eddy-Covariance Data
}

\author{
Yongfeng $\mathrm{Qi}^{1,4} \cdot$ Xiaodong Shang ${ }^{1}$. Guiying $\mathrm{Chen}^{1}$. \\ Zhiqiu $\mathrm{Gao}^{2}$. Xueyan $\mathrm{Bi}^{3}$
}

Published online: 14 December 2015

C) Springer Science+Business Media Dordrecht 2015

\section{Erratum to: Boundary-Layer Meteorol (2015) 157:173-189 DOI 10.1007/s10546-015-0060-6}

Unfortunately, in the original publication the author's (Yongfeng Qi) affiliation has been published incorrectly. The correct version should read as given below:

The online version of the original article can be found under doi:10.1007/s10546-015-0060-6.

$凶$ Xiaodong Shang xdshang@scsio.ac.cn

1 State Key Laboratory of Tropical Oceanography, South China Sea Institute of Oceanology, Chinese Academy of Sciences, Guangzhou 510301, China

2 State Key Laboratory of Atmospheric Boundary Layer Physics and Atmospheric Chemistry, Institute of Atmospheric Physics, Chinese Academy of Sciences, Beijing, China

3 Institute of Tropical and Marine Meteorology, China Meteorological Administration, GuangZhou, China

4 University of Chinese Academy of Sciences, Beijing 100049, China 